\title{
THE EIGENFUNCTIONS OF COMPACT WEIGHTED ENDOMORPHISMS OF $C(X)$
}

\author{
HARALD UHLIG
}

\begin{abstract}
In this note we characterize the eigenmanifolds of compact operators $u C_{\Phi}: f \rightarrow u \cdot f \circ \Phi$ on $C(X)$ and determine their ascents. As an application we show an easy method for computing the eigenmanifolds of a matrix with at most one nonzero element in each row.
\end{abstract}

In the sequel $X$ will always denote a compact Hausdorff space, $u$ a function in $C(X)$, and $\Phi$ a continuous function from $X$ to $X$. Let $\Phi_{n}$ be the $n$th iterate of $\Phi$; i.e., $\Phi_{0}(x)=x$ and $\Phi_{n}(x)=\Phi\left(\Phi_{n-1}(x)\right)$ for $n>0$ and $x \in X$. $c \in X$ is called a fixed point of $\Phi$ of order $n$ if $n$ is a positive integer, $\Phi_{n}(c)=c$, and $\Phi_{k}(c) \neq c$ for $k=1, \ldots, n-1$.

By $u C_{\Phi}$ we denote the operator $u C_{\Phi}: f \rightarrow u \cdot f \circ \Phi$ on $C(X)$. This is a weighted endomorphism, and every weighted endomorphism may be represented in this way (see Kamowitz [1]). Kamowitz [1] proved the following result:

THEOREM A. Suppose $X$ is a compact Hausdorff space, $u$ in $C(X)$, and $\Phi$ a continuous function from $X$ into $X$.

(1) The map $u C_{\Phi}: f \rightarrow u \cdot f \circ \Phi$ is compact iff for each connected component $C$ of $\{x \mid u(x) \neq 0\}$ there exists an open set $V \supset C$ such that $\Phi$ is constant on $V$.

(2) If $u C_{\Phi}$ is compact, then $\sigma\left(u C_{\Phi}\right) \backslash\{0\}=\left\{\lambda \mid \lambda^{n}=u(c) \cdots u\left(\Phi_{n-1}(c)\right)\right.$ for some positive integer $n$ and some fixed point $c$ of $\Phi$ of order $n, \lambda \neq 0\}$.

Our aim here is to characterize the eigenfunctions of a compact $u C_{\Phi}$. To do that we need some more notation: We always assume that $\Phi$ satisfies the conditions of Theorem $\mathrm{A}(1)$ so that $u C_{\Phi}$ is compact. We call $x, y \in X$ equivalent $(x \sim y)$ if there exist $n, m$ in $\{0,1,2, \ldots\}$ so that $\Phi_{n}(x)=y$ and $\Phi_{m}(y)=x$. The equivalence classes are denoted by $[x]$. For any $\lambda$ in $\mathbf{C} \backslash\{0\}$ let $C_{\lambda}:=\{c$ in $X \mid c$ is a fixed point of $\Phi$ of order $n$ for some positive integer $n$ and $\left.\lambda^{n}=u(c) \cdots u\left(\Phi_{n-1}(c)\right)\right\}$. Obviously if $x \sim y$ and $x$ in $C_{\lambda}$, then $y$ is in $C_{\lambda}$, so let $\tilde{C}_{\lambda}:=\left\{[x] \mid x\right.$ is in $\left.C_{\lambda}\right\}$ and $m_{\lambda}$ be the number of equivalent classes in $\tilde{C}_{\lambda} . m_{\lambda}$ is finite by Theorem B and the compactness of $u C_{\Phi}$. For every $c \in C_{\lambda}$ let $h_{c, \lambda}$ denote the following function from $X$ to $\mathbf{C}$ or $\mathbf{R}$ respectively:

$$
h_{c, \lambda}(x):= \begin{cases}\lambda^{-r} u(x) \cdots u\left(\Phi_{r}(x)\right) & \text { for every } r \text { in }\{0,1,2, \ldots\} \text { and } x \in \Phi_{r}^{-1^{\prime}}(\{c\}), \\ 0 & \text { otherwise. }\end{cases}
$$

It is easy to see that $h_{c, \lambda}$ is well defined (remember that e.g. $c$ is in every $\Phi_{k n}^{-1}(\{c\})$ if $c$ is a fixed point of $\Phi$ of order $n$, but then $\left.\lambda^{k n}=u(c) \cdots u\left(\Phi_{k n-1}(c)\right)\right)$. Furthermore

Received by the editors February 19, 1985 and, in revised form, August 26, 1985.

1980 Mathematics Subject Classification. Primary 47B38, 47B05, 46E25.

Key words and phrases. Compact weighted endomorphisms, eigenfunctions, matrices with many zeros. 
$\left\{h_{c_{1}, \lambda}, \ldots, h_{c_{k}, \lambda}\right\}$ is linearly dependent iff, for some $i \neq j, c_{i} \sim c_{j}$. Finally, let $W_{0}:=W:=\{x \mid u(x) \neq 0\}$ and $W_{k}:=\Phi\left(W \cap W_{k-1}\right)$ for $k>0$. For additional notation see Taylor [2].

The principal result of this note is the following theorem.

THEOREM B. (1) Let $\lambda \in \sigma\left(u C_{\Phi}\right) \backslash\{0\}$ and $\left\{c_{1}, \ldots, c_{m_{\lambda}}\right\}$ be representative elements of all equivalence classes in $\tilde{C}_{\lambda}$. Then $\left\{h_{c_{1}, \lambda}, \ldots, h_{c_{m_{\lambda}}, \lambda}\right\}$ is a basis for $\mathcal{N}\left(\lambda-u C_{\Phi}\right)$ and $\alpha\left(\lambda-u C_{\Phi}\right)=1$, where $\alpha\left(\lambda-u C_{\Phi}\right)$ denotes the ascent of $\lambda-u C_{\Phi}$.

(2). The case $\lambda=0$ : If $n>0$, then $\mathcal{N}\left(\left(u C_{\Phi}\right)^{n}\right)=\{f \in C(X) \mid f(x)=0$ for every $\left.x \in W_{n}\right\}$.

Notice that (1) also states that the functions $h_{c, \lambda}$ are continuous.

We will break up the proof by proving several propositions.

Proposition 1. Let $\lambda \in \sigma\left(u C_{\Phi}\right) \backslash\{0\}$. Then $h_{c, \lambda}$ is an eigenfunction for $\lambda$ for every $c \in C_{\lambda}$; that is,

(i) $\lambda h_{c, \lambda}(x)=u(x) h_{c, \lambda}(\Phi(x))$ for all $x \in X$,

(ii) $h_{c, \lambda}$ is continuous.

PROOF. (i) Let $x \in X$. If $x \in \Phi_{r}^{-r}(\{c\})$ for some $r>0$, then

$$
\lambda h_{c}(x)=u(x)\left(\lambda^{-(r-1)} u(\Phi(x)) \cdots u\left(\Phi_{r}(x)\right)\right)=u(x) h_{c, \lambda}(\Phi(x)) .
$$

If $x \notin \Phi_{r}^{-1}(\{c\})$ for every $r \geq 0$, then the same is true for $\Phi(x)$, so $\lambda h_{c, \lambda}(x)=0=$ $u(x) h_{c, \lambda}(\Phi(x))$.

(ii) (1) Since $u$ is continuous, $B=\{x|| u(x)|\geq| \lambda \mid\}$ is compact. As $W$ may be covered with open sets $V_{\beta}$, so that $\Phi$ is constant on each $V_{\beta}, \Phi(B)$ is finite, of cardinality $N$, say. Let $x \in X$ such that $h_{c, \lambda}(x) \neq 0$, and $r$ the minimal number so that $x \in \Phi_{r}^{-1}(\{c\})$. Now $x, \Phi(x), \ldots, \Phi_{r}(x)$ are distinct, whence

$$
\begin{aligned}
\left|h_{c, \lambda}(x)\right| & =|u(x) / \lambda| \cdot|u(\Phi(x)) / \lambda| \cdots\left|u\left(\Phi_{r-1}(x)\right) / \lambda\right| \cdot|u(c)| \\
& \leq \max \left\{1,\left(\|u\|_{\infty} /|\lambda|\right)^{N}\right\} \cdot|u(c)|=: M
\end{aligned}
$$

Therefore $h_{c, \lambda}$ is bounded on $X$.

(2) Let $x \in X$. If $u(x)=0$, then $h_{c, \lambda}(x)=0$ and for every $\varepsilon>0$ there is a neighborhood $U$ of $x$ so that $|u(y)|<\varepsilon|\lambda| / M$ for every $y \in U$. Therefore

$$
\left|h_{c, \lambda}(y)\right|=|\lambda|^{-1}\left|h_{c, \lambda}(\Phi(x))\right||u(y)|<\varepsilon
$$

for every $y \in U$ and thus $h_{c, \lambda}$ is continuous at $x$. If $u(x) \neq 0$, then $\Phi$ is constant on an open neighborhood $U$ of $x$ and therefore

$$
\left|h_{c, \lambda}(x)-h_{c, \lambda}(y)\right|=|\lambda|^{-1}\left|h_{c, \lambda}(\Phi(x))\right||u(x)-u(y)|<\varepsilon
$$

for a suitable neighborhood $U^{\prime} \subset U$ of $x$ and every $y \in U^{\prime}$. So $h_{c, \lambda}$ is continuous.

Proposition 2. Let $\lambda \in \sigma\left(u C_{\Phi}\right), \lambda \neq 0$, and $f$ an eigenfunction for $\lambda$. Then

(i) For every $c \in C_{\lambda}$ there exists $\alpha(c)$ such that $f(x)=\alpha(c) h_{c, \lambda}(x)$ for every $r \geq 0$ and $x \in \Phi_{r}^{-1}(\{c\})$.

(ii) If $x \notin \Phi_{r}^{-1}(\{c\})$ for every $c \in C_{\lambda}$ and $r \geq 0$, then $f(x)=0$.

ProOF. (i) Let $c \in C_{\lambda}$ and $\alpha(c):=f(c) / u(c)$ (remember $\lambda \neq 0$ !). Then for $r \geq 0$ and $x \in \Phi_{r}^{-1}(\{c\})$ we have by iteration

$$
f(x)=\lambda^{-r} u(x) u(\Phi(x)) \cdots u\left(\Phi_{r-1}(x)\right) f\left(\Phi_{r}(x)\right)=\alpha(c) h_{c, \lambda}(x) .
$$


(ii) This part of the proof is actually the same as for Proposition 4 in [1] and is repeated here for the sake of completeness:

Let $x \notin \Phi_{r}^{-1}(\{c\})$ for every $c \in C_{\lambda}, r \geq 0$. If $x$ is a fixed point of $\Phi$, of order $n$, say, then by iteration $f(x)=\lambda^{-n} u(x) \cdots u\left(\Phi_{n-1}(x)\right) f(x)$ and, since $x \notin C_{\lambda}$, we conclude that $f(x)=0$.

If $x \in \Phi_{r}^{-1}(\{c\})$ for some fixed point $c \notin C_{\lambda}$ and $r \geq 1$, then, since $f(c)=0$, we have $f(x)=\lambda^{-r} u(x) \cdots u\left(\Phi_{r-1}(x)\right) f(c)=0$.

Finally, we may suppose that all $\Phi_{r}(x)$ are distinct. Let $\delta:=|\lambda| / 2$. Since $B:=\{x|| u(x) \mid \geq \delta\}$ is compact and by Theorem $\mathrm{A} W$ may be covered by open sets on which $\Phi$ is constant, $\Phi(B)$ is finite, of cardinality $N$, say. Therefore for every $n>N$

$$
\begin{aligned}
|f(x)| & =|u(x) / \lambda||u(\Phi(x)) / \lambda| \cdots\left|u\left(\Phi_{n-1}(x)\right) / \lambda\right|\left|f\left(\Phi_{n}(x)\right)\right| \\
& \leq\left(\|u\|_{\infty} /|\lambda|\right)^{N} 2^{N-n}\|f\|_{\infty} \rightarrow 0 \quad(n \rightarrow \infty) .
\end{aligned}
$$

Thus $f(x)=0$. Q.E.D.

Let $\left\{c_{1}, \ldots, c_{m_{\lambda}}\right\}$ be representative elements of all equivalence classes in $\tilde{C}_{\lambda}$. Then $\left\{h_{c_{1}, \lambda}, \ldots, h_{c_{m_{\lambda}}, \lambda}\right\}$ is a basis for $\mathcal{N}(\lambda-A)$ if $0 \neq \lambda \in \sigma\left(u C_{\Phi}\right)$. So what remains to be done for part (1) of Theorem $B$ is

Proposition 3. Let $0 \neq \lambda \in \sigma\left(u C_{\Phi}\right)$ and $f \in \mathcal{N}\left(\left(\lambda-u C_{\Phi}\right)^{2}\right)$. Then $f \in$ $\mathcal{N}\left(\lambda-u C_{\Phi}\right)$.

PROOF. Since $g:=\left(\lambda-u C_{\Phi}\right) f$ is an eigenfunction for $\lambda$, we know by Proposition 2 that if $x$ is not in $\Phi_{r}^{-1}(\{c\})$ for some $c \in C_{\lambda}$ and $r \geq 0$, then $g(x)=0$. If $c \in C_{\lambda}$ there exists $\alpha(c)$ so that $g(x)=\alpha(c) h_{c, \lambda}(x)$ for every $r \geq 0$ and $x \in \Phi_{r}^{-1}(\{c\})$ by Proposition 2, so we have to show that $\alpha(c)=0$. Let $c$ be of order $n$. Since by iteration

evaluation at $c$ yields

$$
f=n \cdot \frac{g}{\lambda}+\left(u C_{\Phi}\right)^{n} \frac{f}{\lambda^{n}}
$$

$$
f(c)=n \alpha(c) h_{c, \lambda}(c) / \lambda+f(c)
$$

for $g$ is an eigenfunction and $\lambda^{n}=u(c) \cdots u\left(\Phi_{n_{1}}(c)\right)$. Therefore $\alpha(c)=0$.

So far we have proved Theorem B(1). Part (2) follows from

Proposition 4. $\left(u C_{\Phi}\right)^{k} f=0 \Leftrightarrow f(x)=0$ for every $x \in W_{k}$.

ProOF. By induction:

$(\Rightarrow)$ Let $k=1$ and $u C_{\Phi} f=0$. Then for any $x \in W$ we have $0=u(x) f(\Phi(x))$, whence $f(\Phi(x))=0$. If $k>1$ and $\left(u C_{\Phi}\right)^{k} f=0$, we know by induction that $u(x) f(\Phi(x))=0$ for every $x \in W_{k-1}$. Furthermore, if $x \in W$, then $u(x)$. $\neq 0$, so that $f(\Phi(x))=0$. Thus $f$ vanishes on $W_{k}$.

$(\Leftarrow)$ Let $k=1$ and $f(x)=0$ for every $x \in W_{1}$. For $x \in X$ either $x \in W$ and therefore $f(\Phi(x))=0$ or $x \notin W$ and $u(x)=0$. Thus $u C_{\Phi} f=0$. Now let $k>1$ and $f(x)=0$ for every $x \in W_{k}$. We have to show that $u(x) f(\Phi(x))=0$ for every $x \in W_{k-1}$, because then the assertion follows by induction hypothesis. But this is trivial since either $x \notin W$ and $u(x)=0$, or $\Phi(x) \in W_{k}$ and $f(\Phi(x))=0$, if $x \in W_{k-1}$.

EXAMPLE 1. We want to give an example for Theorem $\mathrm{B}(2)$ that the case $\mathcal{N}\left(\left(u C_{\Phi}\right)^{n}\right) \neq \mathcal{N}\left(\left(u C_{\Phi}\right)^{n+1}\right)$ for ever $n$ may occur. Let $X:=\{0\} \cup\{1 / n \mid n \in \mathbf{N}\}$ with the topology induced by the usual topology on $\mathbf{R}$ so that $X$ is compact. Let 
$u(x)=x$ and $\Phi(1 / n)=1 /(n+1), \Phi(0)=0$. These are continuous functions satisfying the conditions of Theorem $\mathrm{A}$. Therefore $u C_{\Phi}$ is a compact operator on $C(X)$, where $C(X)$ may obviously be identified with $c(\mathbf{N}):=\left\{\left(a_{n}\right)_{n \in \mathbf{N}} \mid \lim _{n \rightarrow \infty} a_{n}\right.$ exists $\}$. Since there are no fixed points $c \neq 0$ of $\Phi$ of any order, $\sigma\left(u C_{\Phi}\right)=\{0\}$ by Theorem A. Now $W_{k}=\{x \in X \mid 0<x<1 / k\}$, so $\mathcal{N}\left(\left(u C_{\Phi}\right)^{k}\right)=\left\{\left(a_{n}\right) \mid a_{n}=0\right.$ for every $n>k\}$ and the union of all $\mathcal{N}\left(\left(u C_{\Phi}\right)^{k}\right)$ is exactly the set of all $\left(a_{n}\right)$ satisfying $a_{n}=\mathbf{0}$ for all but finitely many $n$.

EXAMPLE 2. We give an application of our results to the finite-dimensional case. Let $X=\{1, \ldots, n\}$ with the discrete topology. Then $C(X)$ will be identified with $\mathbf{K}^{n}$, where $\mathbf{K}=\mathbf{C}$ or $\mathbf{K}=\mathbf{R}$ is the underlying scalar field. Every linear operator may (and will) be identified with the matrix $\left(a_{i j}\right)_{1 \leq i, j \leq n}$ with $a_{i j}=\left(A \delta_{j}\right)(i)$, where $\delta_{j}(j)=1, \delta_{j}(i)=0$ if $i \neq j$.

$$
\left[\begin{array}{llllll}
0 & 0 & 0 & 0 & 0 & 0 \\
0 & 0 & 0 & 0 & 1 & 0 \\
0 & 0 & 0 & 0 & 0 & 2 \\
3 & 0 & 0 & 0 & 0 & 0 \\
0 & 4 & 0 & 0 & 0 & 0 \\
0 & 5 & 0 & 0 & 0 & 0
\end{array}\right]
$$
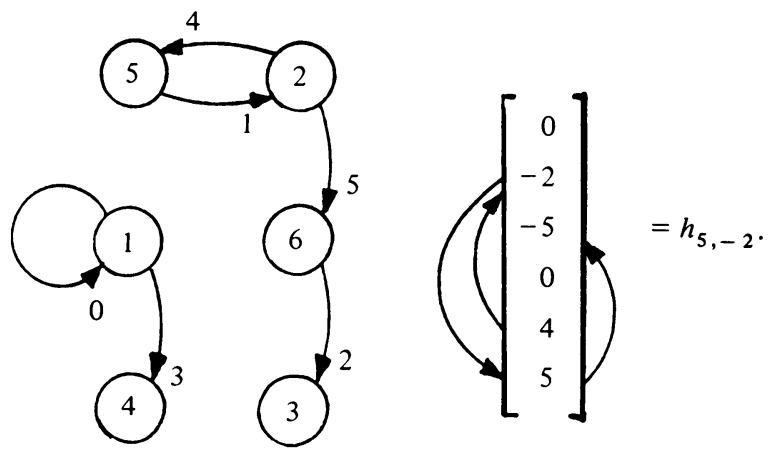

$$
\sigma\left(u C_{\Phi}\right)=\{-2,0,2\}
$$

$\mathcal{N}(A)=\left\{\left(x_{n}\right) \mid x_{1}=x_{2}=x_{5}=x_{6}=0\right\}, \quad \mathcal{N}\left(A^{2}\right)=\left\{\left(x_{n}\right) \mid x_{5}=x_{2}=0\right\}=\mathcal{N}\left(A^{3}\right)$.

If $A=u C_{\Phi}$, then $a_{i j}=u(i)$ if $j=\Phi(i)$ and $a_{i j}=0$ otherwise, so there is at most one nonzero element in each row. Conversely let $A$ have this property. Then for $i=1, \ldots, n$ let $j=\Phi(i)$ and $u(i)=a_{i j}$, if $a_{i j}$ is the unique nonzero element in row $i$. If $a_{i j}=0$ for all $j=1, \ldots, n$ we let $i=\Phi(i)$ and $u(i)=0$. Then obviously $A=u C_{\Phi}$.

Now the eigenvalues and eigenvectors are easily determined: first find out all cycles of $\Phi$. e.g. by drawing $n$ dots with numbers $1, \ldots, n$ and an arrow from dot $j$ to dot $i$ if $\Phi(i)=j$, adding $u(i)$ to that arrow for later purposes. For each cycle multiply all the $u(i)$ of this cycle and calculate the $k$ th roots, where $k$ denotes the number of elements of this cycle: these are all eigenvalues possibly except 0 .

Take one eigenvalue $\lambda \neq 0$ and a cycle corresponding to that $\lambda$. Choose an arbitrary dot $j$, say, of that cycle and set $x_{j}:=u(j)$. Now follow the arrows. If you reach dot $i$ from dot $k$ let $x_{i}$ be the product of $\lambda^{-1} u_{i}$ and $x_{k}$. When you are done with all the dots which belong to the "connected component" containing the cycle set all other $x_{i}=0$. This is an eigenvector for $\lambda$.

If you do this for every cycle corresponding to $\lambda$ you get a basis for the eigenspace $\mathcal{N}(\lambda-A)$.

In order to determine $\mathcal{N}\left(A^{r}\right)$ remove all arrows where $u_{i}=0$. Now $\mathcal{N}(A)$ consists of all $\left(x_{k}\right)$, where $x_{k}=0$ if there is a directed path of length one starting in dot $k$ (to dot $k$ itself or any other dot), and $x_{k}$ is arbitrary otherwise. Similarly for $\mathcal{N}\left(A^{r}\right), r>1$ : "one" has to be replaced by "r" and it is allowed to "use" the same arrow more than one time. 
There is a diagonalization for $A$ iff $\mathcal{N}(A)=\mathcal{N}\left(A^{2}\right)$. Of course all these results are easily obtained by direct verification as well.

\section{REFERENCES}

1. H. Kamowitz, Compact weighted emdomorphisms of $C(X)$, Proc. Amer. Math. Soc. 83 (1981).

2. A. E. Taylor, Introduction to functional analysis, Wiley, New York, 1958.

FACHBEREICH 3 (MATHEMATIK), SEKR. 6-4, TECHNISCHE UNIVERSitÄt BeRLiN, STRASSE DES 17 JUNI 136, D-1000 BERLIN 12, WEST GERMANY

Current address: School of Economics, University of Minnesota, Minneapolis, Minnesota 55455 